\title{
Variability among individuals is generated at the gene expression level
}

\author{
Lloyd S. Peck, ${ }^{1,3}$ Michael A. S. Thorne, ${ }^{1}{ }^{\text {Joseph I. Hoffman, }}{ }^{2}$ Simon A. Morley, ${ }^{1}$ and Melody S. Clark ${ }^{1}$ \\ ${ }^{1}$ British Antarctic Survey, High Cross, Madingley Road, Cambridge CB3 OET United Kingdom \\ ${ }^{2}$ Department of Animal Behaviour, University of Bielefeld, Postfach 100131, 33501 Bielefeld, Germany
}

\begin{abstract}
Selection acts on individuals, specifically on their differences. To understand adaptation and responses to change therefore requires knowledge of how variation is generated and distributed across traits. Variation occurs on different biological scales, from genetic through physiological to morphological, yet it is unclear which of these carries the most variability. For example, if individual variation is mainly generated by differences in gene expression, variability should decrease progressively from coding genes to morphological traits, whereas if post-translational and epigenetic effects increase variation, the opposite should occur. To test these predictions, we compared levels of variation among individuals in various measures of gene expression, physiology (including activity), and morphology in two abundant and geographically widespread Antarctic molluscs, the clam Laternula elliptica and the limpet Nacella concinna. Direct comparisons among traits as diverse as heat shock protein QPCR assays, whole transcription profiles, respiration rates, burying rate, shell length, and ash-free dry mass were made possible through the novel application of an established metric, the Wentworth Scale. In principle, this approach could be extended to analyses of populations, communities, or even entire ecosystems. We found consistently greater variation in gene expression than morphology, with physiological measures falling in between. This suggests that variability is generated at the gene expression level. These findings have important implications for refining current biological models and predictions of how biodiversity may respond to climate change.
\end{abstract}

Key words: AFLP; climate change; evolution; metabolism; phenotypic plasticity; resilience; resistance.

\section{INTRODUCTION}

Natural selection acts upon variation among individuals. Our understanding of variability has improved considerably in recent years, but the integration of variability in biology is poorly developed in many areas. Bennet (1987) highlighted the lack of focus on variability in ecological and physiological research, which he termed the "Tyranny of the Golden Mean." Even now, the vast majority of studies primarily quantify population averages, and models are predominantly designed around average characteristics. Furthermore, there is a large and growing body of work on responses to stress and environmental change that almost exclusively deals with average responses. This approach provides valuable data on underlying mechanisms, but the "average" animal is unlikely to be the best predictor of how species and ecosystems will respond to change. Variability among individuals is of prime importance in shaping responses.

Under changing conditions, such as those produced by current climate change, individuals toward one end of the genetic range of a population will be better suited

Manuscript received 16 April 2014; revised 18 December 2014; accepted 16 January 2015. Corresponding Editor: P. T. Raimondi.

${ }^{3}$ Corresponding author. E-mail: 1spe@bas.ac.uk to the new conditions than the average individual. To understand responses to climate change, we therefore need to characterize individual variability and predict how this may change in the future. Shifts in means of traits in populations can be achieved equally by translocation of the distribution or by the removal of parts of the distribution. The former is adaptational and the latter a consequence of selective deletion, yet the differences are rarely studied and difficult to quantify.

Recent studies have begun to incorporate interindividual variation into ecological models (LloydSmith et al. 2005, Breckling et al. 2006) where, for instance, higher scale phenomena such as swarms, distributions, or trophic networks can be modeled using information on the behavior of individuals. BarbosaMorais et al. (2012) also suggested that phenotypic variation among species may be greater than variation in their protein coding genes. They observed significant differences in alternative splicing complexity between vertebrate lineages and argued this could be a factor contributing to the large phenotypic differences among vertebrate species that have very similar repertoires of protein-coding genes. Some authors have asked how much variation exists within and between populations (e.g., Oleksiak et al. 2002). These studies have posed specific questions relating to variability, but empirical examples are limited and generally focus on a specific 
biological level (e.g., enzyme activity or gene expression). Hence, an important but largely neglected question is "How does variation change across the continuum from gene expression through physiology to morphology?" This is the focus of the current study.

In studies of the evolutionary dynamics of phenotypic traits, fitness has been identified with population genetic variability for over 80 years (Fisher 1930). Quantitative genetic models emphasize the importance of genetic variation in dictating outcomes, for instance in relation to thermal sensitivity during warming (Kingsolver et al. 2004). The majority of studies linking genetic variation with physiological performance are on humans and highly specific, for example showing how genetic sequence variation in genes encoding antioxidant enzymes may alter susceptibility to chronic obstructive pulmonary disease (Tang et al. 2013). However, there are also an increasing number of studies on non-model species showing that variability of individual genes or groups of genes strongly influence physiological performance. For example, two genes (GGA1 and GGA5) explain a significant proportion of variation among individuals in immune responsiveness in chickens (Luo et al. 2014). Polymorphic aryl hydrocarbon receptors are associated with dioxin tolerance in Atlantic killifish (Proestou et al. 2014); and adaptation to local environments and climate change is effected by selection acting on polymorphic loci and variability in gene expression levels (Sørensen et al. 2007, Hoffman and Willi 2008, Pespeni et al. 2011). A small number of empirical studies have compared gene expression and allelic variation with physiological performance, but these emphasize that variation in physiology is related to gene expression (Oleksiak et al. 2002, 2005, Whitehead and Crawford 2005, Hanski and Saccheri 2006, Franks et al. 2007, Clark et al. 2013). There is also often an assumption that differences among individuals are mainly genetically based (Gaston and Spicer 2004). However, several factors complicate the links between genotype and phenotype, including expression regulation, alternative splicing, epigenetic effects, post translational modifications, with further complexity added when considering the dynamics of genetics, demography and evolution when studying population level responses to external factors such as the accumulation of damage following environmental insult or stress (Tomanek 2011, Shaw and Etterson 2012). This leads to questions of how much variability exists in these different processes, how they are related, and how they contribute to phenotypic variability.

If individual variation is mainly generated at the gene level it might be expected that, within a homogenous population, variation would decrease with biological scale, from gene expression to physiology and morphology. Factors working in this direction would include that large numbers of genes contribute to physiological processes and the overall physiological state, and at any one time it is likely that some will be at high levels of expression while others are reduced. The outcome at the higher scale would be lower levels of variation between individuals. However, if post translational and epigenetic effects, and higher level factors such as cytosol composition and environmental fluctuation, increase variation then the opposite pattern would emerge. To understand how variation varies from gene expression to morphology firstly requires a method for comparing variation at different scales. Variation is often assessed as total range, or as a measure of the spread of distributions such as standard deviations or standard error. These metrics, however, depend on the units used; the magnitude of the metric and values need to be standardized before comparisons can be made across metrics.

Here we apply the scale independent and unitless Wentworth Scale (Krumbein 1936) for the first time to our knowledge to evaluate these predictions by comparing variability among individuals at different levels of biological organization. Thus, inter-individual variation in the expression of candidate genes and transcriptional profiling are compared with standard physiological measures of metabolic rate, activity, upper lethal temperature limits, and morphometric analyses of growth rates and animal size (linear dimensions and mass) in two Antarctic marine molluscs, the soft-shell clam Laternula elliptica, and the limpet Nacella concinna. These species both have wide geographic distributions, are locally abundant, amenable to animal husbandry, and similar metrics can be used on both. The choice of two mollusc species reduces phylogenetic differences, while providing an ecological contrast between a sessile infaunal species (L. elliptica) and a mobile grazer ( $N$. concinna). These two species have also been studied extensively in relation to their resistance to environmental change where, for example, juvenile $L$. elliptica react differently to adults to hypoxic stress (Clark et al. 2013), tenacity in limpets has a very different relation to temperature than most biological functions (Morley et al. 2012), and acclimation in $N$. concinna, among other Antarctic marine invertebrates was shown to take months to achieve rather than days or weeks (Peck et al. 2014). N. concinna and L. elliptica are both important components of an ecosystem on the west coast of the Antarctic Peninsula experiencing among the fastest regional warming globally.

\section{Methods}

\section{Animal collections}

Approximately 50 individuals of each species were collected by scuba divers at 10-20 $\mathrm{m}$ depth near the British Antarctic Survey station on Rothera Point, Adelaide Island, Antarctica $\left(67^{\circ} 34^{\prime} 07^{\prime \prime} \mathrm{S}, 68^{\circ} 07^{\prime} 30^{\prime \prime} \mathrm{W}\right)$ in January 2009. A restricted size range (medium to large adults: $3.01-10.33 \mathrm{~g}$ ash-free dry mass [AFDM] for L. elliptica and 0.07-0.39 g AFDM for $N$. concinna) was used in both species to minimize the effects of developmental stage, age, and reproductive maturity. 
All animals were genetically analyzed, as described subsequently, and assessed for respiration rate, activity level, and a range of morphometric measures. However, constitutive gene expression could not be analyzed for animals in upper temperature limit (UTL) trials. Half of these were therefore screened for UTL and the other half for gene expression.

\section{Baseline data}

The analyses of variation among individuals presented here are meta-analyses of respiration rate, activity, gene expression, UTL, morphometric measures, and age. Each of these characters was evaluated for at least 25 individuals of each species.

\section{Respiration rate}

After collection, animals were held for $2-3$ weeks in an aquarium with a 12-h light: 12 -h dark light regime, within $0.5^{\circ} \mathrm{C}$ of ambient temperature $\left(0.7^{\circ}-1.1^{\circ} \mathrm{C}\right)$ to allow for recovery from any collection stress and to ensure specimens were in a standard metabolic state, excluding the effects of feeding, which can take up to 3 weeks in Antarctic marine species (Peck 1998). They were not fed during this period, although very small amounts of food may have been available for $N$. concinna grazing on biofilms and for L. elliptica from any microalgae passing through the aquarium intake filtration. No feces were produced after two weeks of holding specimens prior to measurements. Individuals were then transferred underwater to glass or acrylic respirometers $\left(\sim 1.65 \mathrm{~L}\right.$ or $4.6 \mathrm{~L}$ for L. elliptica; $80 \mathrm{~cm}^{3}$ or $235 \mathrm{~cm}^{3}$ for $N$. concinna) and oxygen consumption measured using closed-bottle methods similar to Obermuller et al. (2010). Oxygen concentration was monitored using a Fibox-3 fiber optic sensor (PreSens $\mathrm{GmbH}$, Regensburg, Germany) and was not allowed to fall below $80 \%$ saturation during $4-10 \mathrm{~h}$ experiments. Animal volumes were measured after trials by displacement and respirometer volume adjusted accordingly. Oxygen consumption was measured as the change in concentration and converted to $\mu \mathrm{mol} \mathrm{O}_{2} / \mathrm{g}$ ash-free dray mass (AFDM) for use in later comparisons.

\section{Activity}

In L. elliptica, activity was measured as reburying rate (BRI) after removal from sediment following Peck et al. (2004). Specimens were placed on fine sand in temperature-controlled tanks at $0.0^{\circ} \mathrm{C}$ and allowed to bury. Video recordings (Panasonic AG6124HB time-lapse recorder; Panasonic, Kadoma, Japan) were subsequently analyzed using a JVCBR-S610E video analysis machine (JVC Kenwood, Yokohama, Japan). Times to complete reburial were recorded. In $N$. concinna, activity was assessed as the duration of attachment (tenacity) while resisting a set force, following Morley et al. (2011). Preliminary trials showed that a mass of $200 \mathrm{~g}$ attached to the shell via a pulley system resulted in tenacities of $0.5-60 \mathrm{~min}\left(\right.$ at $\left.0^{\circ} \mathrm{C}\right)$.

\section{Temperature limits}

UTLs were measured for each individual on one-half of the specimens of each species. Specimens were warmed at $1{ }^{\circ} \mathrm{C} / \mathrm{d}$ until they no longer responded to external stimuli, following Peck et al. (2009). When animals were close to their UTL, $N$. concinna were tested for responsiveness by touching the exposed foot or cephalic tentacles and L.elliptica by touching the siphon and mantle margins with a blunt probe. The temperature where individuals failed to respond to these stimuli was recorded as the UTL.

\section{Tissue sampling for molecular analyses}

Tissue samples $(\sim 100 \mathrm{mg})$ were taken from all specimens after other procedures had been completed and stored in $96 \%$ ethanol for amplified fragment length polymorphism (AFLP) analysis. Twenty-five animals were used in the UTL experiments and the remainder of the animals was sampled for gene expression analyses. For gene expression analyses, foot tissue was taken from $N$. concinna and mantle and siphon tissue from $L$. elliptica and flash frozen in liquid nitrogen and extracted using the standard TRI reagent (TriSure; Bioline, London, UK) method according to manufacturer's instructions. A 1- $\mu \mathrm{g}$ aliquot of total RNA was DNAse treated using 0.4U DNase I (Ambion, Grand Island, New York, USA) in $10 \mathrm{mmol} / \mathrm{L}$ DTT per $100 \mathrm{mmol} / \mathrm{L}$ $\mathrm{MgCl}_{2}$ buffer and reverse transcribed using a first strand synthesis kit (Promega, Madison, Wisconsin, USA) for Q-PCR.

\section{Gene expression}

Constitutive gene expression was evaluated for the heat shock protein (HSP) genes Hsp70A and Hsp70B from foot samples in $N$. concinna $(n=24$ samples $)$ and in the mantle and siphon of L. elliptica ( $n=24$ samples). QPCR of the HSP genes (Hsp70A and Hsp70B) was conducted following Clark et al. (2008) with $\beta$-actin used as the control sequence. In brief, HSP and actin sequences were amplified Brilliant $\mathrm{SYBR}^{\circledast}$ Green QPCR Master Mix (Stratagene, La Jolla, California, USA) and an MX3000P (Stratagene). PCR conditions were as follows: $95^{\circ} \mathrm{C}$ for 10 minutes, 40 cycles of $95^{\circ} \mathrm{C}$ for 30 seconds, $60^{\circ} \mathrm{C}$ for 1 minute, and $72^{\circ} \mathrm{C}$ for 1 minute with a final dissociation curve step as per manufacturer's recommendations. All amplifications were carried out with a specific HSP primer pair and an actin control primer set on each plate and in triplicate. Each primer set was checked to ensure that no primer dimers were produced during the course of the amplification reaction and the $\beta$ actin was checked between individuals and experiments to ensure homogeneity of expression and suitability as a control. For each primer set, $R^{2}$ values and PCR efficiencies were checked over a fourfold $10 \times$ dilution series and the values calculated using the MxPro-MX3000P v 3.00 Build 311 Schema 74 software (Stratagene) with the $\mathrm{Ct}(\mathrm{dR})$ values exported into Microsoft Excel (Microsoft, Redmond, Washington, 
USA; Clark et al. 2008). Relative expression ratios were derived using the Relative Expression Software Tool (REST) (Pfaffl et al. 2002) except, in this experiment, comparative expression levels were related to a single individual as all the animals were effectively "untreated" (software available online). ${ }^{4}$ The 24 individuals of $L$. elliptica for gene expression analysis were also subjected to tagged 454 sequencing following Clark et al. (2010). Briefly, the raw data was screened for adaptor sequences and other artefacts of the pyrosequencing procedure using Crossmatch (P. Green, personal communication) and also vector sequences using the UniVec database (available online). ${ }^{5}$ Following this, 778629 sequences were entered into the Newbler program (454 Life Sciences, Branford, Connecticut, USA) for assembly. This resulted in 18290 contigs that were used as the transcriptome backbone. All the individual reads were then mapped onto this assembly using Newbler with default parameters. Files containing the reads are available from the National Center for Biotechnology Information (NCBI; see Data Availability). Hsp70A transcripts were present in all individuals, allowing variation among individuals to be determined based on normalized values. An additional pool of 305 transcripts present in all individuals was identified and assessed for variability of expression.

DNA extraction, AFLP genotyping, and genetic analysis

AFLPs were developed to test for cryptic population structure as, if present, this could introduce an additional scale of variability into our analyses. Total genomic DNA was extracted from a small piece of tissue using Qiagen's DNeasy tissue extraction kit (Hilden, Germany) using the manufacturer's recommended protocols. The AFLP protocol was that used by Hoffman et al. (2010) and employed the selective primer combinations in (Appendix: Table A1). To test for genetic homogeneity of individuals within each species, Bayesian cluster analyses of the AFLP genotype data sets using STRUCTURE 2.2.3 were conducted (Pritchard et al. 2000). This program uses a maximum likelihood approach to cluster individuals into $K$ populations without prior knowledge of population membership and partitions the data set in such a way that any departures from Hardy-Weinberg and linkage equilibrium within the resulting clusters are minimized. The estimated $\log$ probability of the data, denoted $\ln P(D)$, is calculated for each value of $K$, allowing estimation of the most likely number of populations. Five independent runs were conducted for $K=1-5$ (where 1 represents a lack of population structure, and 5 represents five genetically distinct sub-populations) using $1 \times 10^{6}$ Markov chain Monte Carlo iterations after a burn-in of $1 \times 10^{5}$, specifying the correlated allele frequencies model and assuming admixture.

\footnotetext{
${ }^{4}$ www.gene-quantification.de/rest.html

${ }^{5}$ www.ncbi.nlm.nih.gov/VecScreen/UniVec.html
}

\section{Morphometrics and age}

Shell length, width, and height $( \pm 0.1 \mathrm{~mm})$ were measured in all individuals of both species. The tissues and shells were then separated and the shells air dried to constant mass and weighed. Tissues were dried for $48 \mathrm{~h}$ at $60^{\circ} \mathrm{C}$, cooled in a desiccator, and weighed. Samples were then ignited in a muffle furnace at $475^{\circ} \mathrm{C}$, cooled in a desiccator, and the remaining ash weighed. AFDM was obtained by subtraction. Masses of samples taken for genetic analyses were noted and dry mass and AFDM corrected accordingly. Age was obtained from annual shell growth checks only for L. elliptica. External growth checks were too indistinct for $N$. concinna to be aged.

\section{Quantifying variation}

Superficial comparisons of variation between characters can be made by analyzing maximum:minimum ratios or ratios of first and third quartiles, with the latter less vulnerable to extreme values. These ratios define the spread of a data set but are not useful for comparisons across diverse data sets. A method for comparing variability at different scales was developed by Krumbein (1936) for analyzing grain size in sediments, that he termed the Wentworth Scale. Initially the first to third quartile ratio $(\mathrm{Q} 3: \mathrm{Q} 1)$ is calculated. The square root of this value is calculated and the $\log _{2}$ of the resulting value taken: $\left.\log _{2} \sqrt{\mathrm{Q} 3: \mathrm{Q} 1}\right)$. This produces an arithmetic scale of variation on which highly diverse data can be compared. A Wentworth grade of 0.1 compared to another of 0.8 means that variation in the latter is eight times greater than in the former for any two metrics.

\section{Data analyses}

Correlation between the measures was assessed using variance inflation factors (VIF) (Zuur et al. 2010). VIF indicates which variables have their variance increased due to co-linearity with other variables in a regression model. The term with the highest VIF was sequentially dropped and the analysis re-run to investigate correlations between the remaining variables. Although the cutoff for strong correlation is subject to debate, iterations continued until all the values had a VIF less than 10 (Kutner et al. 2004).

\section{Results}

\section{Genetic analyses}

All $N$. concinna individuals were genotyped at seven selective primer combinations producing 202 loci, of which 160 (79.2\%) were polymorphic (Appendix: Table A1). L. elliptica individuals were genotyped at six selective combinations, yielding 92 loci, of which 70 (76.1\%) were polymorphic (Table A1). Seven L. elliptica failed to amplify at one or more primer combination and were excluded from subsequent analyses. Population structure was tested using the STRUCTURE program. Highly concordant log likelihood values were obtained 
TABLE 1. Measures taken of Laternula elliptica, with the ratio of the maximum to minimum (max : $\min$ ), third quartile to first quartile $(\mathrm{Q} 3: \mathrm{Q} 1)$, and their respective Wentworth Scale value.

\begin{tabular}{lccccc}
\hline \hline \multicolumn{1}{c}{ Measure } & Max: Min & Q3: Q1 & $\sqrt{\mathrm{Q} 3: \mathrm{Q} 1}$ & $\begin{array}{c}\text { Wentworth } \\
\text { Scale value }\end{array}$ & $\begin{array}{c}\text { Type of } \\
\text { measure }\end{array}$ \\
\hline ManHSP70A & 30 & 2.875 & 1.695 & 0.761 & $\mathrm{G}$ \\
ManHSP70B & 24 & 3.153 & 1.775 & 0.828 & $\mathrm{G}$ \\
SiphHSP70A & 14.5 & 1.950 & 1.396 & 0.481 & $\mathrm{G}$ \\
SiphHSP70B & 65 & 3.571 & 1.889 & 0.918 & $\mathrm{G}$ \\
454 ManHSP70A & 11.02 & 2.486 & 1.577 & 0.657 & $\mathrm{G}$ \\
O2 consumption & & & & & \\
$\quad$ Whole animal & 3.311 & 1.397 & 1.182 & 0.241 & $\mathrm{P}$ \\
Per unit AFDM & 9.685 & 2.280 & 1.510 & 0.594 & $\mathrm{P}$ \\
Per unit DM & 8.879 & 2.187 & 1.479 & 0.564 & $\mathrm{P}$ \\
Burial duration & 11.508 & 2.052 & 1.432 & 0.518 & $\mathrm{P}$ \\
BRI & 21.60 & 2.128 & 1.458 & 0.544 & $\mathrm{P}$ \\
UTL & 1.370 & 1.083 & 1.040 & 0.057 & $\mathrm{P}$ \\
Age & 2.333 & 1.285 & 1.133 & 0.181 & $\mathrm{M}$ \\
Tissue DM & 4.812 & 1.742 & 1.319 & 0.400 & $\mathrm{M}$ \\
AFDM & 5.318 & 1.772 & 1.331 & 0.413 & $\mathrm{M}$ \\
Shell DM & 4.332 & 1.482 & 1.217 & 0.284 & $\mathrm{M}$ \\
Shell length & 1.684 & 1.172 & 1.082 & 0.115 & $\mathrm{M}$ \\
\hline
\end{tabular}

Notes: Type of measure refers to metric type: gene expression, G; physiology, P; morphology, M. Abbreviations are Man, Mantle; Siph, Siphon; DM, dry mass; AFDM, ash-free dry mass; BRI, burrowing rate index; UTL, upper temperature limit.

for each set of replicates (Fig. A1). For both species, the highest values were consistently associated with $K=1$, indicating a lack of population structure, and hence individuals sampled for each species were from homogenous populations.

\section{Individual variation}

Frequency distributions for each of the gene expression, physiological, and morphometric variables did not differ significantly from normality (Anderson-Darling tests, all $P>0.05$ ). Animals of similar sizes (shell length varied between 21.0 and $31.1 \mathrm{~mm}$ in $N$. concinna and between 50.1 and $85.0 \mathrm{~mm}$ in L. elliptica) were selected for these analyses and no strong correlations were found among characteristics such as activity and oxygen consumption (Tables A2 and A3). The only strong correlations were found between morphometric measures, including tissue dry mass (TDM) and AFDM $(\mathrm{VIF}=60-213)$ and length and width $(\mathrm{VIF}=7.9-17.2)$. After sequentially removing variables with the highest VIF values, little change was obtained in any of the other metrics except between the heat shock proteins (HSP) Hsp70A and Hsp70B in L. elliptica, (VIFs $=11.2$ and 12.16, respectively, when TDM was included; Table A2). This suggests that body size affected Hsp70 expression, even within this restricted size range.

\section{Wentworth Scale analyses}

Wentworth Scale values for L. elliptica ranged from 0.06 for UTL to 0.92 for Hsp70B gene expression levels in the siphon, measured using QPCR. Variation in siphon Hsp70B expression level in L. elliptica was 15.33 times greater than variation in UTL. In this species, the highest four values were all obtained for the expression of HSP genes, suggesting that levels of gene expression are more variable than the other measures. This was also supported by a genome-wide analysis of the digital gene expression data, in which only $305(1.7 \%)$ of 18290 contigs were expressed in all 24 individuals. Wentworth Scale values for each of these genes ranged from 0.20 1.89 (Fig. A9). The distribution of values was right skewed with a median around 0.6. The value for Hsp70B of 0.66 was close to the median, indicating that HSP gene variability is representative of most genes, and supports their use in the QPCR analysis here. Following variability in gene expression level, the next most variable metrics were mass specific metabolic rate at 0.56 and $0.59\left(\mathrm{O}_{2}\right.$ consumed/g dry mass or AFDM, respectively) and activity (BRI) at 0.52 . Morphological measures varied least (Table 1).

Similar results were obtained for $N$. concinna. One exception was that the highest Wentworth grade was obtained for tenacity (0.99), which was $\sim 25$ times higher than variation in UTL (Table A2). Highthroughput gene expression data were not available for $N$. concinna, but QPCR measures of expression level of the HSP genes again had among the highest Wentworth values at 0.68 (Hsp70A) and 0.65 (Hsp70B), and UTL again had the lowest value. As with L. elliptica, morphological measures yielded consistently lower Wentworth grades.

Most of the measures made here fall into three rough categories: gene expression, physiology (including activity, metabolic rate, and UTL), and morphology (age, mass measures, and linear dimensions; Tables 1 and 2). When the three categories are compared for L. elliptica, average Wentworth grades for gene expression were higher than physiological and morphological measures but only the difference between gene expression and morphological measures was significant (Fig. 1). A 
TABLE 2. Measures taken of Nacella concinna, with the ratio of the maximum to minimum, third quartile to first quartile, and their respective Wentworth Scale value.

\begin{tabular}{lccccc}
\hline \hline \multicolumn{1}{c}{ Measure } & Max: Min & Q3:Q1 & $\sqrt{\mathrm{Q} 3: \mathrm{Q} 1}$ & $\begin{array}{c}\text { Wentworth } \\
\text { Scale value }\end{array}$ & $\begin{array}{c}\text { Type of } \\
\text { measure }\end{array}$ \\
\hline HSP70B & 20.50 & 2.466 & 1.570 & 0.651 & $\mathrm{G}$ \\
$\mathrm{O}_{2}$ consumption & & & & & \\
Whole animal & 25.319 & 2.034 & 1.426 & 0.512 & $\mathrm{P}$ \\
Per unit AFDM & 72.054 & 2.466 & 1.570 & 0.651 & $\mathrm{P}$ \\
Per unit DM & 69.326 & 2.339 & 1.529 & 0.613 & $\mathrm{P}$ \\
Tenacity & 1253.5 & 3.943 & 1.986 & 0.989 & $\mathrm{P}$ \\
UTL & 1.278 & 1.056 & 1.028 & 0.397 & $\mathrm{P}$ \\
Length & 1.490 & 1.213 & 1.101 & 0.139 & $\mathrm{M}$ \\
Width & 1.528 & 1.213 & 1.101 & 0.139 & $\mathrm{M}$ \\
Height & 2.400 & 1.266 & 1.125 & 0.170 & $\mathrm{M}$ \\
Tissue DM & 5.963 & 1.653 & 1.286 & 0.363 & $\mathrm{M}$ \\
AFDM & 6.589 & 1.610 & 1.269 & 0.344 & $\mathrm{M}$ \\
Shell dry mass & 7.015 & 1.645 & 1.283 & 0.359 & $\mathrm{M}$ \\
\hline
\end{tabular}

similar pattern was found for $N$. concinna, where variability was consistently highest for measures of gene expression level, intermediate for physiology, and lowest for morphological traits (Fig. 1). As for L. elliptica, only the difference between gene expression level and morphology was statistically significant (Fig. 1).

\section{Discussion}

Selection acts on individuals, specifically on their differences. To understand adaptation and responses to environmental change therefore requires knowledge of how inter-individual variation is generated and distributed across different traits. However, this requires a robust metric of variation, which is independent of units or scale of measurement, in order to compare data of different types. Here we have applied an established metric in a novel way to compare biological variability from genes, through metabolism, activity, and UTL. The Wentworth Scale is dimensionless, independent of sample size when samples are representative of the underlying distribution, and allows direct comparison of distributions of any variable, as the ratio of Wentworth Scale values is a direct ratio of the variability of two metrics (Krumbein 1936). In our case, the variables of interest range from gene expression through physiological to morphological measures. We have compared measures across individuals, but our approach could, in principle, be used to compare variability at any scale, such as populations, assemblages, or even entire ecosystems. Such methods are essential for the develop-
FIG. 1. Box and whisker plots for averaged Wentworth grades for L. elliptica, and $N$. concinna. Each plot contains Wentworth data for gene expression, activity (includes data for activity and metabolism [oxygen consumption]), and morphological measures. For each metric shown, the thick bar represents the median value, the box represents the first and third quartiles, and whiskers represent total range. Whiskers for $N$. concinna physiology and gene expression are close to quartile ranges and not visible on this plot. All data used to calculate mean values were derived from Tables 1 and 2. For both species, differences between values for gene expression and physiology were not significant. For $N$. concinna, variation in physiology was not significantly higher than morphology measures, but gene expression data were (physiology vs. morphology, $t=-2.01, P=0.11$; gene expression vs. morphology, $t=-9.95, P=2.2 \times 10^{-5}$ ). For L. elliptica, variation in physiology was not significantly different from morphological measures $(t=1.33, P=0.22)$, but gene expression values were $(t=4.75, P=0.002)$.
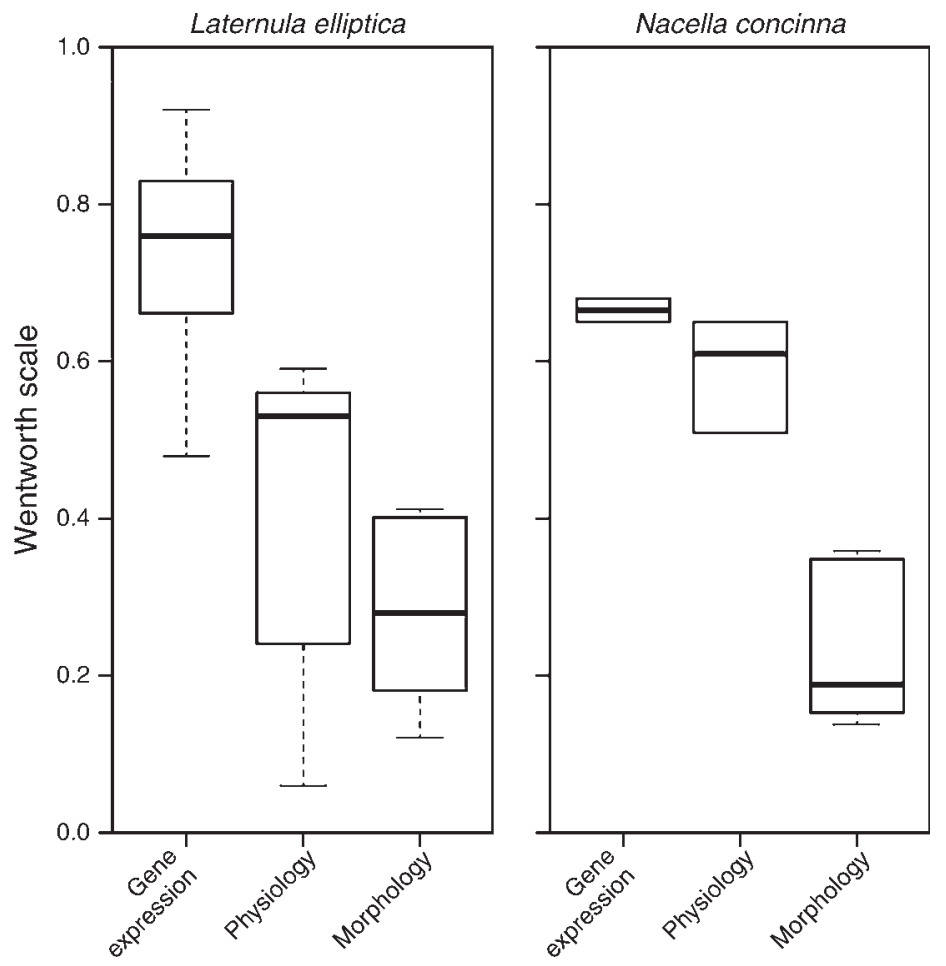
ment of the systems based analyses toward which biologists are increasingly being drawn.

\section{How and where is individual variability generated?}

The first questions to address in this type of study are (1) how is variability generated, and (2) where in the continuum from genes to ecosystems is that variability greatest? Genes and gene expression are at the smallest physical scale in biology and since Fisher (1930) defined population fitness in terms of genetic variability many authors have provided evidence that genetic variation is important in generating phenotypic variation (e.g., Koehn and Hilbish 1987, Thurston et al. 2002). Whitehead and Crawford (2005) stated that "Widespread heritable variation combined with extensive natural variation in gene expression ... provides the substrate for evolution," but few data exist to support or refute these statements. Recently global genome-wide association studies are providing evidence of complex genotype-phenotype interactions (Rosenberg 2011). These studies are at a scale not yet possible in nonmodel species, but they provide an indication of variability across scales in a highly specialized biological system, the inferences of which are directly relevant to ecological systems.

It has often been assumed that inter-individual variation depends primarily on differences in the genetic code (Gaston and Spicer 2004). However, environmental factors also have effects, and more recently epigenetic factors (e.g., methylation) have been shown to be heritable (e.g., Slatkin 2009). In addition, efforts to develop predictive systems biology models using both experimental and theoretical network approaches are revealing the complexity of factors that influence biochemical pathways, even at the single cell level, producing measureable intracellular fluctuations that lead to higher-level effects (Elowitz et al. 2002, Rosenfeld et al. 2005). For example biochemical parameters that can be accurately described in vitro using MichaelisMenten parameters are not well described in vivo, but have been shown to be affected by microscale intracellular states such as metabolic fluxes, concentration control coefficients, and cell history (Klipp et al. 2004, Ruess et al. 2013). These all potentially add layers where variation may be affected. The data in this study for variability at the molecular level are for gene expression and not for protein coding differences. Although there have been conflicting reports, methodological improvements and the ability to survey increasingly large numbers of transcripts/proteins in the same experiment is increasingly showing a positive correlation between expression levels of orthologous proteins and mRNAs (Rees et al. 2011), with $40 \%$ of the variance in protein abundance explained by mRNA levels (Schwanhäusser et al. 2011). This indicates that gene expression variation feeds into variation at the cellular level (Little et al. 2008).
The data presented here support the contention that much of the variability among individuals of a given species has a genetic basis, albeit via differences in gene expression as opposed to coding differences. In both $L$. elliptica and $N$. concinna, the greatest overall variability occurred in gene expression metrics, with physiological metrics intermediate and morphological traits least variable (Fig. 1), although differences between gene expression and physiological metrics were not statistically significant. Only two genes were measured here using Q-PCR, and it may be that an expanded QPCR survey of gene expression would show different levels of variability between individuals. However, this seems highly unlikely as the 454 pyrosequencing data clearly indicate that the genes analyzed by Q-PCR were broadly representative of the 305 genes shared between all $L$. elliptica individuals in our study (Fig. A8). These 305 genes represented only $1.7 \%$ of the genes identified across all individuals in the 454 analysis, indicating large inter-individual variation in the genes being expressed at that point in time. Expression levels themselves were also highly variable among individuals.

There was large individual variation in gene expression. Thus there was no correlation between the level of expression of any given gene among individuals. For example, the most variable transcript (a cytosolic actin sequence, which was different from the actin sequence used in the Q-PCR analysis) showed the highest expression in individual 6 and the lowest in individual 9, whereas the Mnk map kinase, which in the combined transcriptome showed the highest expression levels, showed most expression in individual 13 and the lowest in individual 1 (Fig. 2), which emphasizes the complexity of genic interactions. Q-PCR and 454 are different ways of quantifying gene expression. Measured expression levels of the same transcript varied to a limited extent depending on the method used, as shown by our comparative data for Hsp70A using both Q-PCR and 454 sequencing. However, when the Wentworth scale was applied to the 454 data, the results were consistent with those based solely on the Q-PCR data (minimum = 0.2 and maximum $=1.89$ ). As shown in Fig. A8, the QPCR based value for Hsp70A was 0.66 , very close to the distribution mode of the 454 data. This is important because it validates the use of this gene in $N$. concinna, for which 454 data were lacking. Although not presented within a broader comparative framework, other studies have shown similar levels of variation in gene expression among individuals. For example, in a microarray study of cardiac gene expression in the killifish Fundulus heteroclitus collected from different natural populations but raised in a common environment, $94 \%$ of the transcripts varied significantly among individuals (Oleksiak et al. 2005), and an earlier study on the same species showed more variation in gene expression between individuals than between populations (Oleksiak et al. 2005). By implication, the pattern identified here of declining variability from gene expression to the whole 
animal may continue to population and community levels, or even higher scales.

Our data indicate that the variation generated at the gene expression level is not translated through to whole animal responses, as measured by UTL or morphological characteristics. This pattern has been seen in some previous studies where specific traits, such as cellular energy budgets and isolated peripheral nerve function, perform over wider temperature ranges and fail at much higher temperatures than the whole animal (Mark et al. 2005, Young et al. 2006), and stress resistance in animals responds hierarchically from cells through tissues to whole animals (Pörtner et al. 2007). Ecological studies are usually concerned with whole-animal and population-level responses, i.e., the overall performance of an individual or population. Understanding how a given phenotype can resist or adapt under changing conditions depends on how much variability is present at different biological levels to contribute to this flexibility. Studies on a limited gene set examining myogenesis in teleost fish have shown that some genes contributing to muscle development are far more plastic in their response to environmental conditions than others and therefore contribute more under altered conditions (Fernandes et al. 2005, 2007, Johnston 2006). Selection driven by changing conditions does not act on all genes equally. Identifying where the greatest variability lies provides a starting point to understanding and quantifying the complex environment-genotype interactions that ultimately determine whether a species survives or fails in a changing world.

\section{Differences between species}

When we compared specific characters, rather than groups of characters, the most variable characters in $N$. concinna were activity (tenacity), oxygen consumption, and gene expression (Table A3). In L. elliptica, the same sets of characters were also the most variable, but in the reverse order. These findings can potentially be explained by the differences in the types of activity and the life style of the animals. Tenacity and reburial are both metrics of activity, but the mechanisms they employ are different; reburial involves repeated foot muscle contraction and hydraulic pumping to fluidize vicinal sediment (Peck et al. 2004), and is an aerobic activity. Tenacity in $N$. concinna is achieved by isometric muscle tension, limited by muscle fatigue and not oxygen supply. The two activities thus do not invoke the same biochemical and physiological processes. With regard to oxygen consumption, L. elliptica is infaunal and sedentary, and might be expected to have a lower metabolic rate compared with the mobile $N$. concinna.

Despite these differences, when levels of inter-individual variability were analyzed after grouping the measures into three categories (gene expression, physiology, and morphology), remarkably similar results were obtained for the two species. This was initially somewhat surprising given that they differ in a variety of ecological and life history traits. For example, the mobile grazing $N$. concinna has the potential for dispersal via attachment to macroalgae broken from the seabed via storms or ice impacts not available to the sedentary suspension feeding L. elliptica. These factors should reduce gene flow in L. elliptica, meaning that isolation and genetic drift might be expected to have eroded standing genetic variation compared to $N$. concinna. No such signal was present in our gene expression data. Furthermore $N$. concinna is a broadcast spawner with a long pelagic phase (Bowden et al. 2009) and L. elliptica has protected development for extended periods, which might suggest there is little, if any, pelagic dispersal (Peck et al. 2007). However, Bosch and Pearse (1988) reported encapsulated L. elliptica eggs suspended in the water column and Harper (2012) reported an absence of genetic differentiation at the population level for sites separated by $100 \mathrm{~km}$, indicating effective dispersal occurs in this species.

\section{Response to environmental change}

Selection works on differences between individuals allowing populations to adapt to change. There is little information for most species on levels of variability in populations for key characteristics conferring resistance to change, or indeed what these characteristics are. We also know little of how rapidly those characteristics can be altered through phenotypic plasticity, gene flow between populations or mutation, which generates new genetic variants (Peck 2011). Many early genetic studies showed selection often favors balanced combinations, Galton's "mediocrity of the mean" (Mather 1973). In this context the "average" animal is not necessarily optimally suited to their environment at any given time, but as the environment is rarely static, the optimal adaptation changes over time, meaning that an "average" animal may do best over extended periods, especially as in many cases, variation in fitness will have to be maintained and accumulated over multiple years and annual bouts of survival and reproduction (Shaw and Etterson 2012). However, environmental change can occur rapidly enough to produce strong directional selection (c.f. Umina et al. 2005, Franks et al. 2007, Korves et al. 2007), and this is likely to be the case with current and projected climate change.

Selection due to environmental change may reduce variation by removing part of the normal distribution for any character, with the new distribution being concatenated, as has been shown in rapid evolution selection experiments (Breckels et al. 2014). This moves the mean of the character measured, and alters its variation. Such changes in character means (genetic, physiological, or morphological) in populations may not indicate an adaptive response, but may merely reflect the loss of part of the population's variation. It is important when interpreting responses to environmental change to understand the underlying variability in the population, how this may have been altered and also whether this 


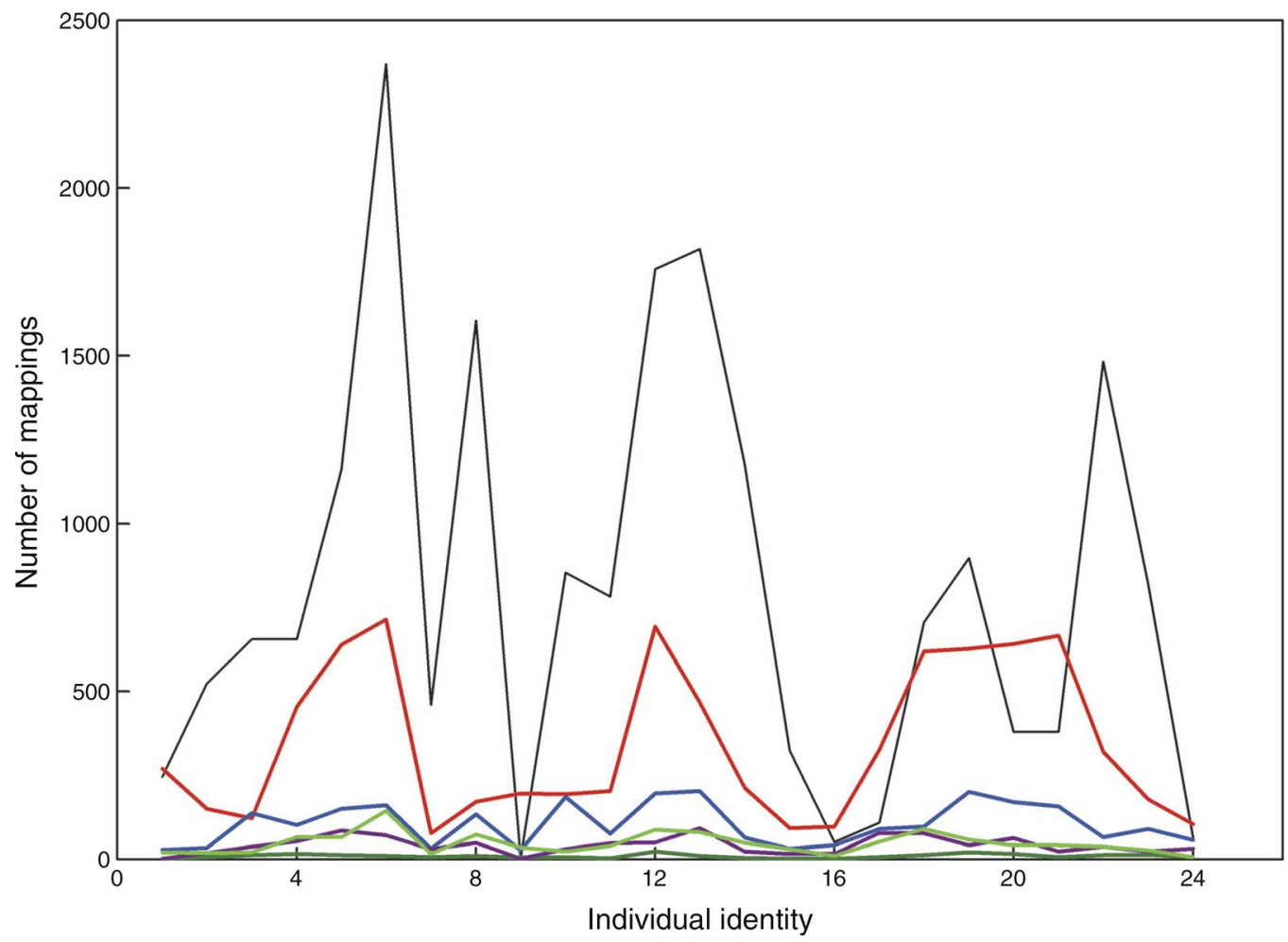

FIG. 2. Example showing variability in the number of 454 reads mapping onto six transcripts, representing different gene functions, in each of the $24 \mathrm{~L}$. elliptica analyzed. The number of 454 reads varied between individuals, so the mappings were normalized to the total number of reads, thus ensuring proportionality between individuals. Each transcript had an associated contig identifier and a putative function assigned based on sequence similarity searching. Black, contig01069, actin (cytoskeletal); red, contig17382, unknown function; blue, contig14497, ferritin (antioxidant); dark green, contig02493, HSP70 (chaperone); purple, contig00447, Mnk Map kinase interacting serine/threonine protein kinase (potential role in response to environmental stress); light green, contig00046, glyceraldehyde 3-phosphate dehydrogenase (glycolysis).

change is heritable or the result of a plastic response to the challenge (Potvin and Tousignant 1996, Hoffman and Willi 2008, Hoffman and Sgrò 2011). Physiological and ecological studies have traditionally covered many species, and next-generation sequencing is now providing the potential to survey gene expression across species, termed comparative genomics. Our analysis using the Wentworth metric indicates that variation is greatest at the gene expression level. Climate change is currently one of the main drivers of long-term environmental change. Extending our approach from individuals to populations and species from different regions and across time offers the tantalizing possibility of elucidating the effects of climate change on variability within a taxon, or even community, and this will help identify the most vulnerable groups. The challenge is to understand how the different measures connect and influence outcomes.

In conclusion, understanding how variability changes across biological levels and how this variability between levels is related is essential for integrating metrics at a variety of scales, which in turn may facilitate understanding of how a variety of phenomena, ranging from those studied here to higher levels (e.g., predator-prey interac- tions and further on to food webs), may respond to environmental change. We employed a metric previously developed for comparing sediment grain sizes to draw biological comparisons. This allowed direct comparison of variation in characteristics not previously possible both within and between species. Our findings indicate that variability is generated at the gene expression level, which has important implications for refining current ecological analyses and input into models and predictions of how biodiversity may respond to change.

\section{ACKNOWLEDGMENTS}

We thank the Diving Team at Rothera Research Station for assistance in collecting specimens. We also thank the marine biologist and assistant at Rothera Research station for technical assistance in running experiments. Support for diving was also supplied by the NERC Diving Facility at the Scottish Association for Marine Science, Oban. The science here was supported through core Natural Environment Research Council funding to the British Antarctic Survey. We are grateful to two unknown referees whose input markedly improved the final paper.

\section{Literature Cited}

Barbosa-Morais, N. L., et al. 2012. The evolutionary landscape of alternative splicing in vertebrate species. Science 338:15871593. 
Bennet, A. F. 1987. Interindividual variability: an underutilised resource. Pages 147-169 in M. E. Feder, A. F. Bennet, W. W. Burggren, and R. B. Huey, editors. New directions in ecological physiology. Cambridge University Press, Cambridge, UK.

Bosch, I., and J. S. Pearse. 1988. Seasonal pelagic development and juvenile recruitment of the bivalve Laternula elliptica in McMurdo Sound, Antarctica. American Zoologist 28:A89.

Bowden, D. A., A. Clarke, and L. S. Peck. 2009. Seasonal variation in the diversity and abundance of pelagic larvae of Antarctic benthic marine invertebrates. Marine Biology 156: 2033-2047.

Breckels, R. D., S. R. Garner, and B. D. Neff. 2014. Rapid evolution in response to increased temperature maintains population viability despite genetic erosion in a tropical ectotherm. Evolutionary Ecology 28:141-155.

Breckling, B., U. Middlehof, and H. Reuter. 2006. Individual based models as tools for ecological theory and application: understanding the emergence of organismal properties in ecological systems. Ecological Modelling 194:102-113.

Clark, M. S., K. P. P. Fraser, and L. S. Peck. 2008. Antarctic marine molluscs do have an Hsp70 heat shock response. Cell Stress and Chaperones 13:39-49.

Clark, M. S., et al. 2010. Insights into shell deposition in the Antarctic bivalve Laternula elliptica. BMC Genomics 11:362.

Clark, M. S., G. Husmann, M. A. S. Thorne, G. Burns, M. Truebano, L. S. Peck, D. Abele, and E. E. R. Phillip. 2013. Hypoxia impacts large adults first: consequences in a warming world. Global Change Biology. http://dx.doi.org/ $10.1111 /$ gcb. 12197

Elowitz, M. B., A. J. Levine, E. D. Siggia, and P. S. Swain. 2002. Stochastic gene expression in a single cell. Science 297 : 1183-1186.

Fernandes, J. M., M. G. MacKenzie, J. R. Kinghorn, and I. A. Johnston. 2005. Functional genomic approaches to investigating developmental plasticity to temperature in the model pufferfish species (Takifugu rubripes). Comparative Biochemistry and Physiology A-Molecular \& Integrative Physiology 141:S349-S350.

Fernandes, J. M., M. G. MacKenzie, J. R. Kinghorn, and I. A. Johnston. 2007. FoxK1 splice variants show developmental stage-specific plasticity of expression with temperature in the tiger pufferfish. Journal of Experimental Biology 210:34613472.

Fisher, R. A. 1930. The genetical theory of natural selection. Oxford University Press, Oxford, UK.

Franks, S. J., S. Sim, and A. E. Weis. 2007. Rapid evolution of flowering time by an annual plant in response to a climate fluctuation. Proceedings of the National Academy of Sciences USA 104:1278-1282.

Gaston, K. J., and J. I. Spicer. 2004. Biodiversity: an introduction. Second edition. Blackwell Publishing, Malden, Massachusetts, USA.

Hanski, I., and I. Saccheri. 2006. Molecular-level variation affects population growth in a butterfly metapopulation. PLoS Biology 4:e129.

Harper, E. M. 2012. Iceberg scour and shell damage in the Antarctic bivalve Laternula elliptica. PLoS ONE 7:e46341.

Hoffmann, A. A., and C. M. Sgrò. 2011. Climate change and evolutionary adaptation. Nature 470:479-485.

Hoffmann, A. A., and Y. Willi. 2008. Detecting genetic response to environmental change. Nature Reviews Genetics 9:421-432.

Hoffman, J. I., et al. 2010. No evidence for genetic differentiation between Antarctic limpet Nacella concinna morphotypes. Marine Biology 157:765-778.

Johnston, I. A. 2006. Environment and plasticity of myogenesis in teleost fish. Journal of Experimental Biology 209:22492264.

Kingsolver, J. G., G. J. Ragland, and J. G. Schlichta. 2004. Quantitative genetics of continuous reaction norms: thermal sensitivity of caterpillar growth rates. Evolution 58:15211529.

Klipp, E., W. Liebermeister, and C. Wierling. 2004. Inferring dynamic properties of biochemical reaction networks from structural knowledge. Genome Informatics 15:125-137.

Koehn, R. K., and T. J. Hilbish. 1987. The adaptive importance of genetic variation. American Scientist 75:134-141.

Korves, T. M., K. J. Schmid, A. L. Caicedo, C. Mays, J. R. Stinchcombe, D. Purugganan, and J. Schmitt. 2007. Fitness effects associated with the major flowering time gene FRIGIDA in Arabodopsis thaliana in the field. American Naturalist 169:E141-E157.

Krumbein, W. C. 1936. The use of quartile measures in describing and comparing sediments. American Journal of Science 32:98-111.

Kutner, M., C. Nachtsheim, and J. Neter. 2004. Applied linear regression models. Fourth edition. McGraw-Hill/Irwin, Boston, Massachusetts, USA.

Little, P. F. R., R. B. H. Williams, and M. R. Wilkins. 2008. Inter-individual variation in expression: a missing link in biomarker biology? Trends in Biotechnology 27:5-10.

Lloyd-Smith, J. O., S. J. Schreiber, P. E. Kopp, and W. M. Getz. 2005. Superspreading and the effect of individual variation on disease emergence. Nature 438:355-359.

Luo, C. L., H. Qu, J. Ma, J. Wang, X. X. Hu, N. Li, and D. M. Shu. 2014. A genome-wide association study identifies major loci affecting the immune response against infectious bronchitis virus in chicken. Infection Genetics and Evolution 21:351-358.

Mark, F. C., T. Hirse, and H. O. Portner. 2005. Thermal sensitivity of cellular energy budgets in some Antarctic fish hepatocytes. Polar Biology 28:805-814.

Mather, K. 1973. Genetical structure of populations. Chapman and Hall, London, UK.

Morley, S. A., et al. 2011. Duration tenacity: a method for assessing acclimatory capacity of the Antarctic limpet, Nacella concinna. Journal of Experimental Marine Biology and Ecology 399:39-42.

Morley, S. A., S. M. Martin, R. W. Day, J. Ericson, C.-H. Lai, M. Lamare, K.-S. Tan, M. A. S. Thorne, and L. S. Peck. 2012. Thermal reaction norms and the scale of temperature variation: latitudinal vulnerability of intertidal nacellid limpets to climate change. PLoS ONE 7(12):e52818.

Obermuller, B., L. S. Peck, D. K. A. Barnes, and S. A. Morley. 2010. Seasonal physiology of Antarctic marine benthic predators and scavengers. Marine Ecology Progress Series 415:109-126.

Oleksiak, M. F., G. A. Churchill, and D. L. Crawford. 2002. Variation in gene expression within and among natural populations. Nature Genetics 32:261-266.

Oleksiak, M. F., J. L. Roach, and D. L. Crawford. 2005. Natural variation in cardiac metabolism and gene expression in Fundulus heteroclitus. Nature Genetics 37:67-72.

Peck, L. S. 1998. Feeding, metabolism and metabolic scope in Antarctic ectotherms. Society for Experimental Biology Seminar Series 66:365-389.

Peck, L. S. 2011. Organisms and responses to environmental change. Marine Genomics 4:237-243.

Peck, L. S., A. D. Ansell, K. E. Webb, L. Hepburn, and M. Burrows. 2004. Movements and burrowing activity in the Antarctic bivalve molluscs Laternula elliptica and Yoldia eightsi. Polar Biology 27:357-367.

Peck, L. S., M. S. Clark, S. A. Morley, A. Massey, and H. Rossetti. 2009. Animal temperature limits and ecological relevance: effects of size, activity and rates of change. Functional Ecology 23:248-253.

Peck, L. S., S. A. Morley, J. Richard, and M. S. Clark. 2014. Acclimation and thermal tolerance in Antarctic marine ectotherms. Journal of Experimental Biology 217:16-22.

Peck, L. S., D. K. Powell, and P. A. Tyler. 2007. Very slow development in two Antarctic bivalve molluses, the infaunal 
clam, Laternula elliptica and the scallop Adamussium colbecki. Marine Biology 150:1191-1197.

Pespeni, M. H., D. A. Garfield, M. K. Manier, and S. R. Palumbi. 2011. Genome-wide polymorphisms show unexpected targets of natural selection. Proceedings of the Royal Society B 279:1412-1420.

Pfaffl, M. W., G. W. Horgan, and L. Dempfle. 2002. Relative expression software tool (REST) for group-wise comparison and statistical analysis of relative expression results in realtime PCR. Nucleic Acids Research 30:1-10.

Pörtner, H. O., L. S. Peck, and G. N. Somero. 2007. Thermal limits and adaptation in marine Antarctic ectotherms: an integrative view. Philosophical Transactions of the Royal Society of London B 362:2233-2258.

Potvin, C., and D. Tousignant. 1996. Evolutionary consequences of simulated global change: genetic adaptation or adaptive phenotypic plasticity. Oecologia 108:683-693.

Pritchard, J. K., M. Stephens, and P. Donnelly. 2000. Inference of population structure using multilocus genotype data. Genetics 155:945-959.

Proestou, D. A., P. Flight, D. Champlin, and D. Nacci. 2014. Targeted approach to identify genetic loci associated with evolved dioxin tolerance in Atlantic killifish (Fundulus heteroclitus). BMC Evolutionary Biology 14:7.

Rees, B. B., T. Andacht, E. Skripnikova, and D. L. Crawford. 2011. Population proteomics: quantitative variation within and among populations in cardiac protein expression. Molecular Biology and Evolution 28:1271-1279.

Rosenberg, N. A. 2011. A population-genetic perspective on the similarities and differences among worldwide human populations. Human Biology 83:659-684.

Rosenfeld, N., J. W. Young, U. Alon, P. S. Swain, and M. B. Elowitz. 2005. Gene regulation at the single-cell level. Science 307:1962-1965.

Ruess, J., A. Milias-Argeitis, and J. Lygeros. 2013. Designing experiments to understand the variability in biochemical reaction networks. Journal of the Royal Society Interface 10:20130588.
Schwanhäusser, B., B. Busse, N. Li, G. Dittmar, J. Schurchhardt, J. Wolf, W. Chen, and M. Selbach. 2011. Global quantification of mammalian gene control. Nature 473:337342.

Shaw, R. G., and J. R. Etterson. 2012. Rapid climate change and the rate of adaptation: insight from experimental quantitative genetics. New Phytologist 195:752-765.

Slatkin, M. 2009. Epigenetic inheritance and the missing heritability problem. Genetics 182:845-850.

Sørensen, J. G., M. M. Nielsen, and V. Loeschcke. 2007. Gene expression profile analysis of Drosophila melanogaster selected for resistance to environmental stressors. Journal of Evolutionary Biology 20:1624-1636.

Tang, S., et al. 2013. Increased cytokines response in patients with tuberculosis complicated with chronic obstructive pulmonary disease. PLoS ONE 8(4):e62385.

Thurston, L., P. F. Watson, and W. V. Holt. 2002. Semen cryopreservation: a genetic explanation for species and individual variation? Cryo Letters 23:255-262.

Tomanek, L. 2011. Environmental proteomics: changes in the proteome of marine organisms in response to environmental stress, pollutants, infection, symbiosis, and development. Annual Review of Marine Science 3:14.1-14.27.

Umina, P. A., A. R. Weeks, R. R. Kearney, S. W. McKechnie, and A. A. Hoffmann. 2005. A rapid shift in a classic clinal pattern in Drosophila reflecting climate change. Science 308: 691-693.

Whitehead, A., and D. L. Crawford. 2005. Variation in tissuespecific gene expression among natural populations. Genome Biology 6:R13.

Young, J. S., L. S. Peck, and T. Matheson. 2006. The effects of temperature on peripheral neuronal function in eurythermal and stenothermal crustaceans. Journal of Experimental Biology 209:1976-1987.

Zuur, A. F., E. N. Ieno, and C. S. Elphick. 2010. A protocol for data exploration to avoid common statistical problems. Methods in Ecology and Evolution 1:3-14.

\section{Supplemental Material}

\section{Ecological Archives}

The Appendix is available online: http://dx.doi.org/10.1890/14-0726.1.sm

\section{Data Availability}

Data associated with this paper have been archived at the National Center for Biotechnology Information, accession number SRA011054: http://www.ncbi.nlm.nih.gov/sra/?term=SRA011054 